\title{
Mg Doped-ZnO Nanoparticle Film as the Interlayer between ZnO Electron Transport Layer and InP Quantum-Dot Layer for Light-Emitting Diodes
}

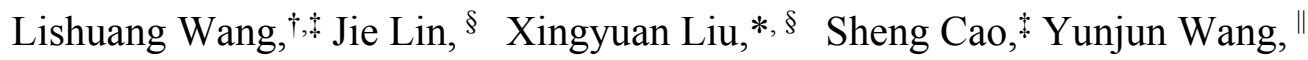 \\ Jialong Zhao, ${ }^{*, \star}$ Bingsuo Zou*,\$ \\ $\dagger$ College of Chemistry and Chemical Engineering, Guangxi University, Nanning 530004, China. \\ * Nano and Energy Research Center, School of Physics Science and Technology; Key Lab of \\ Featured Metal Resources Utilization and Advanced Materials Development, Guangxi University, \\ Nanning 530004, China. \\ $\S$ State Key Laboratory of Luminescence and Applications, Changchun Institute of Optics, Fine \\ Mechanics and Physics, Chinese Academy of Sciences, Changchun 130033, Jilin, China. \\ "| Suzhou Xingshuo Nanotech Co., Ltd. (Mesolight), Suzhou 215123, China.
}

\section{Experimental}

\section{Synthesis of InP/ZnSe/ZnS QDs}

Stock solution for Se precursor: The selenium precursor solution (2M) was prepared by hearing a mixture of selenium $(7.9 \mathrm{~g}, 100 \mathrm{mmol})$ and TOP $(50 \mathrm{~mL})$ to $150^{\circ} \mathrm{C}$ under nitrogen. Stock solution for $\mathbf{S}$ precursor: The sulfur precursor solution (2M) was prepared by hearing a mixture of sulfur $(1.28 \mathrm{~g}, 40 \mathrm{mmol})$ and TOP $(20 \mathrm{~mL})$ to $150^{\circ} \mathrm{C}$ under nitrogen.

Stock solution for $0.4 \mathrm{M}$ Zn precursor(1): A mixture of $\mathrm{ZnO}$ (4.0 g, $50 \mathrm{mmol}$ ), oleic acid 
(70 mL), and $55 \mathrm{~mL}$ ODE was loaded into a $250 \mathrm{~mL}$ three-necked flask and heated to $280^{\circ} \mathrm{C}$ under nitrogen to obtain a colorless, clear solution.

Stock solution for $0.25 \mathrm{M} Z \mathbf{Z n}$ precursor(2): A mixture of $\mathrm{ZnO}(5.1 \mathrm{~g}, 62.5 \mathrm{mmol})$, stearic acid $(130 \mathrm{~mL})$, and $120 \mathrm{~mL}$ ODE was loaded into a $500 \mathrm{~mL}$ three-necked flask and heated to $280^{\circ} \mathrm{C}$ under nitrogen to obtain a colorless, clear solution.

\section{Synthesis of InP/ZnSe/ZnS QDs:}

Typically, $0.45 \mathrm{~g}$ of $\mathrm{InCl}_{3}, 1.2 \mathrm{~g}$ of $\mathrm{ZnCl}_{2}$ and $20 \mathrm{~mL}$ oleylamine were added to a flask, and the mixture was heated to $120 \sim 180^{\circ} \mathrm{C}$ under Ar flow until a clear solution formed. Then the reaction mixture was heated to $200 \sim 220^{\circ} \mathrm{C}$. At this temperature, $2 \mathrm{~mL}(\mathrm{DEA})_{3} \mathrm{P}$ solution was injected into the reaction flask. After 10 60 min, a certain amount ( $4 \mathrm{~mL}$ ) of the as-prepared stock solution for Se precursor (2 M) and $100 \mathrm{~mL}$ stock solution for Zn precursor(2) (0.25 M) were added and the reaction were kept for 30 120 min. At this temperature, another $15 \mathrm{~mL}$ of the as-prepared stock solution for Se precursor (2 M) was swiftly injected into the reaction flask. The reaction mixture was then heated to $280 \sim 320^{\circ} \mathrm{C}$ and $30 \mathrm{~mL}$ stock solution for $\mathrm{Zn}$ precursor(1) $(0.4 \mathrm{M})$ and $3 \mathrm{~mL}$ DDT were added and kept for more than $30 \mathrm{~min}$. At the end, the heating was removed to stop the reaction and allow the flask to cool to room temperature. All manipulations were performed using standard air-free techniques.

\section{Synthesis of ZnO NPs.}

The $\mathrm{ZnO}$ NPs were prepared according to the method reported in the literature ${ }^{1-2}$ with some modification. Dissolving potassium hydroxide $(0.74 \mathrm{~g})$ in methanol $(30 \mathrm{ml})$ was added dropwisely into zinc acetate dihydrate $(1.48 \mathrm{~g})$ in methanol $(60 \mathrm{ml})$ under vigorous stirring. The reaction mixture was stirred at $63{ }^{\circ} \mathrm{C}$ for over $3.5 \mathrm{~h}$. The 
reaction mixture was then cooled to room temperature and the solvent was removed from the precipitate. The precipitate was washed several times with methanol and separated by centrifugation. Then the particles were dissolved in normal butanol at a desirable concentration for experiments.

\section{Synthesis of Mg-doped ZnO NPs.}

Colloidal Mg-doped $\mathrm{ZnO}$ NPs were synthesized by the reported method under ambient conditions with some modification ${ }^{3}$. For a typical synthesis of the $\mathrm{Zn}_{0.85} \mathrm{Mg}_{0.15} \mathrm{O}$ solution, an ethanol solution of potassium hydroxide $(0.4 \mathrm{mmol})$ was dropwise added into a three-neck flask, in which $17 \mathrm{mmol}$ zinc acetate dehydrate and $3 \mathrm{mmol}$ magnesium acetate were dissolved in a mixture of $120 \mathrm{~mL}$ dimethyl sulfoxide and $120 \mathrm{~mL}$ ethanol previously. The solution was kept stirring vigorously for 3 hours after the end of dropwise addition, and then Mg-doped $\mathrm{ZnO}$ NPs were washed and redisperse by using ethyl acetate and ethanol, respectively.

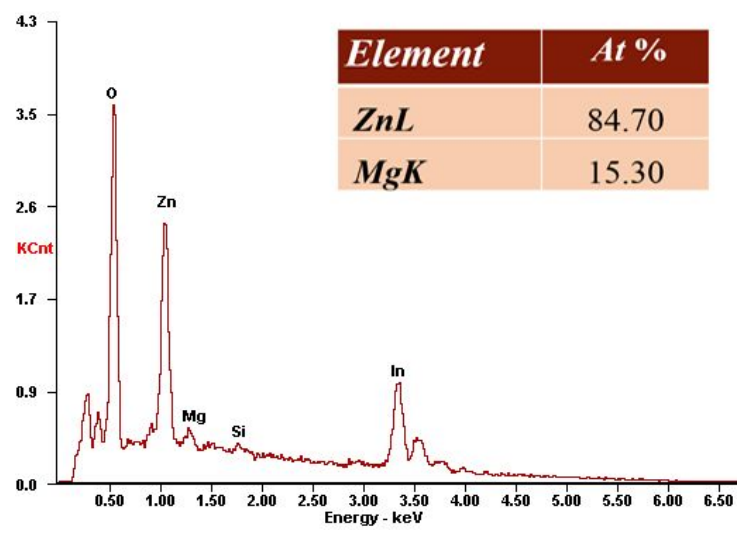

Figure S1. The EDX spectrum of $\mathrm{ZnMgO} \mathrm{NP}$ film.

The composition of $\mathrm{Mg}$ in $\mathrm{ZnO}$ NPs was researched by energy dispersive analysis of X-ray (EDX). The tested sample is glass/ITO/ZnMgO NPs, the 
peak of indium element was came from the ITO substrate. As shown in Figure S1, mass fraction of doped percentage of $\mathrm{Mg}$ in $\mathrm{ZnO} \mathrm{NPs}$ is $\sim 6 \%$, and atom fraction of $\mathrm{Mg}$ in $\mathrm{ZnO}$ NPs is $15 \%$, so the used interlayer is the $\mathrm{Zn}_{0.85} \mathrm{Mg}_{0.15} \mathrm{O}$ NP film.

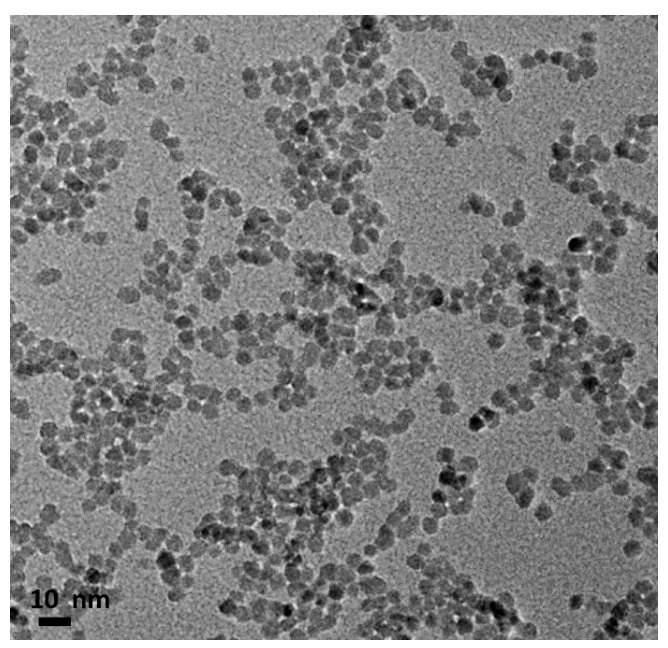

Figure S2. TEM image Mg-doped $\mathrm{ZnO}$ nanoparticles.

Table S1. Fitted results of PL exponential decay of red QDs.

\begin{tabular}{|l|c|c|c|c|}
\hline & $\mathrm{A}_{1}$ & $\begin{array}{c}\tau_{1} \\
(\mathrm{~ns})\end{array}$ & $\mathrm{A}_{2}$ & $\begin{array}{c}\tau_{2} \\
(\mathrm{~ns})\end{array}$ \\
\hline QDs in solution & 0.65347 & 26.97676 & 0.33784 & 72.2233 \\
\hline QDs on solid state & 0.85811 & 25.78771 & 0.14222 & 85.98459 \\
\hline
\end{tabular}

The decay of PL intensity of QDs can be fitted with a double exponential formula:

$$
y=y_{0}+A_{1} e^{-x / t_{1}}+A_{2} e^{-x / t_{2}}
$$

And avenge lifetime is calculate from the following equation:

$$
\tau_{\mathrm{avg}}=\frac{\sum_{i=1}^{n} A_{i} \tau_{i}^{2}}{\sum_{i=1}^{n} A_{i} \tau_{i}}
$$



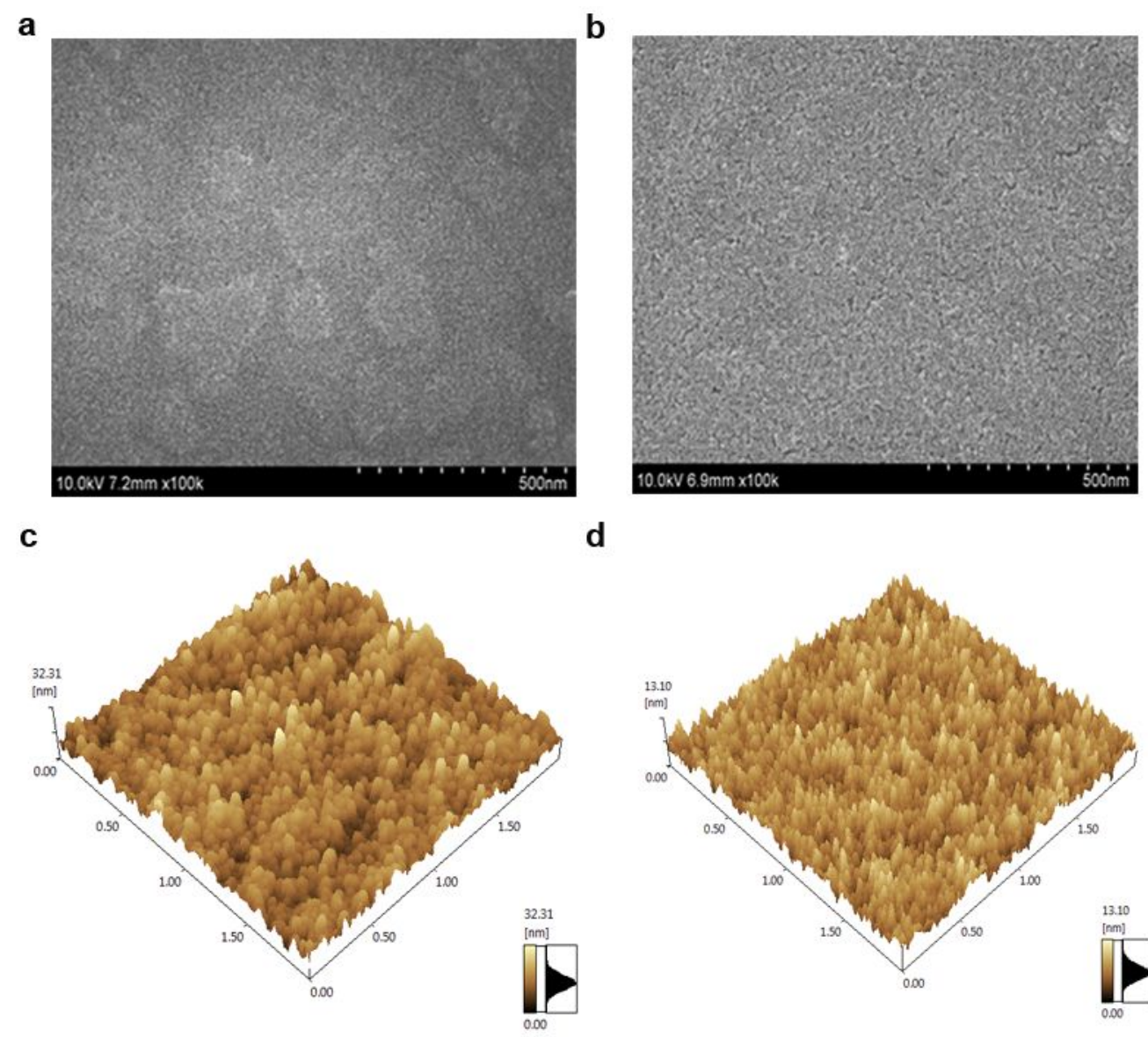

d

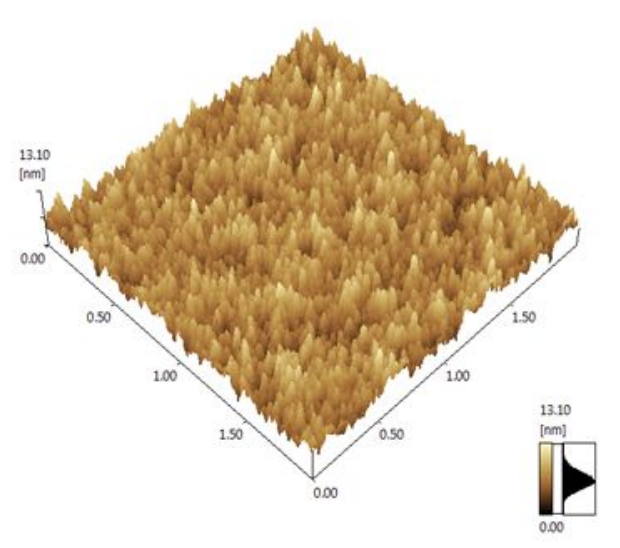

Figure S3. SEM images of NPs on glass (a) ZnO and (b) ZnMgO. 3D AFM images (c) $\mathrm{ZnO}$ NPs and (d) ZnMgO NPs on glass.

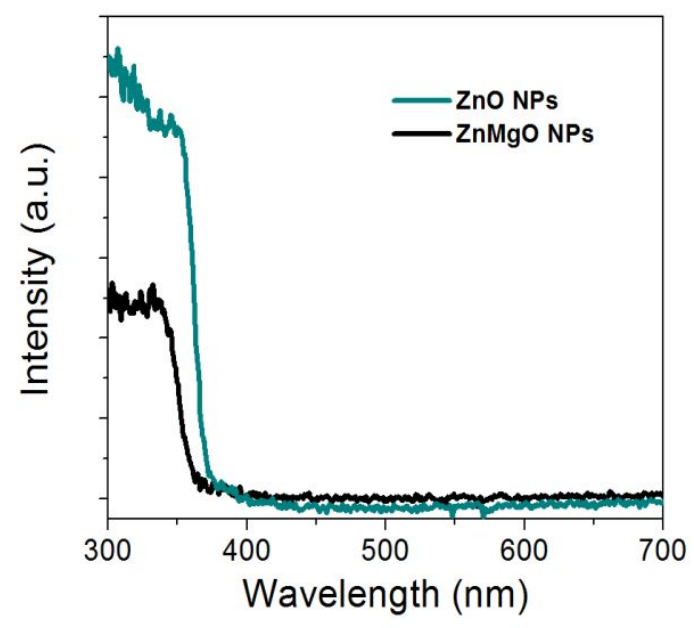

Figure S4. UV-abs spectroscopy of $\mathrm{ZnO}$ and $\mathrm{ZnMgO}$ NPs. 
Table S2. Fitted results of PL exponential decay of different samples, glass/ZnO NPs/QDs (Sample 1), and glass/ZnO NPs/ZnMgO NPs/QDs (Sample 2).

\begin{tabular}{|l|c|c|c|c|}
\hline & $\mathrm{A}_{1}$ & $\begin{array}{c}\tau_{1} \\
(\mathrm{~ns})\end{array}$ & $\mathrm{A}_{2}$ & $\begin{array}{c}\tau_{2} \\
(\mathrm{~ns})\end{array}$ \\
\hline Sample 1 & 0.36055 & 20.28616 & 0.63161 & 5.2136 \\
\hline Sample 2 & 0.28695 & 29.09431 & 0.71814 & 8.25419 \\
\hline
\end{tabular}
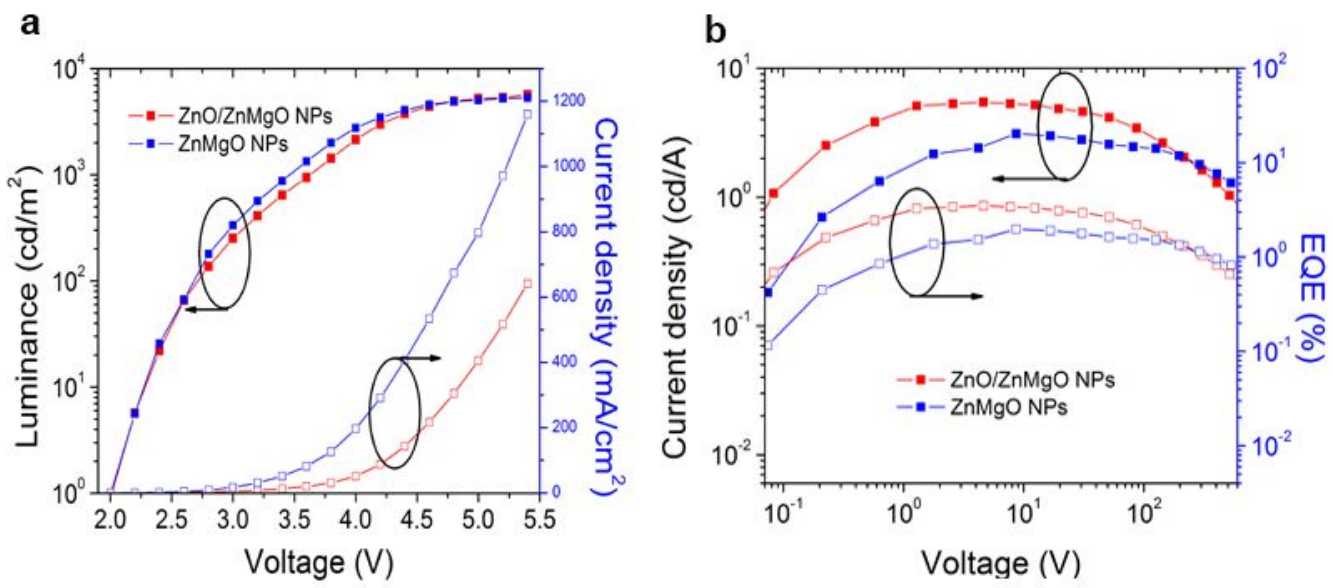

Figure S5. EL performances of red QLEDs with different ETL ( $\mathrm{ZnO} / \mathrm{ZnMgO} \mathrm{NPs}$ or

$\mathrm{ZnMgO} \mathrm{NPs}$ ). (a) Luminance-voltage-current density (L-V-J) characteristics of QLEDs. (b) CE properties and EQE properties as a function of current density.

Table S3. Summary of maximum luminance $\left(\mathrm{L}_{\max }\right)$, maximum $\mathrm{CE}\left(\mathrm{CE}_{\max }\right)$, maximum $\mathrm{EQE}\left(\mathrm{EQE}_{\max }\right)$ of red device with different ETL.

\begin{tabular}{cccc}
\hline Device & $\mathbf{L}_{\max }$ & $\mathbf{C E}_{\mathbf{m a x}}$ & $\mathbf{E Q E}_{\mathbf{m a x}}$ \\
& $\left(\mathbf{c d} / \mathbf{m}^{\mathbf{2}}\right)$ & $\mathbf{( c d / A )}$ & $\mathbf{( \% )}$ \\
\hline $\mathrm{ZnO} / \mathrm{ZnMgO} \mathrm{NPs}$ & 5680 & 5.46 & 3.49 \\
$\mathrm{ZnMgO} \mathrm{NPs}$ & 5300 & 3.10 & 2.00 \\
\hline
\end{tabular}




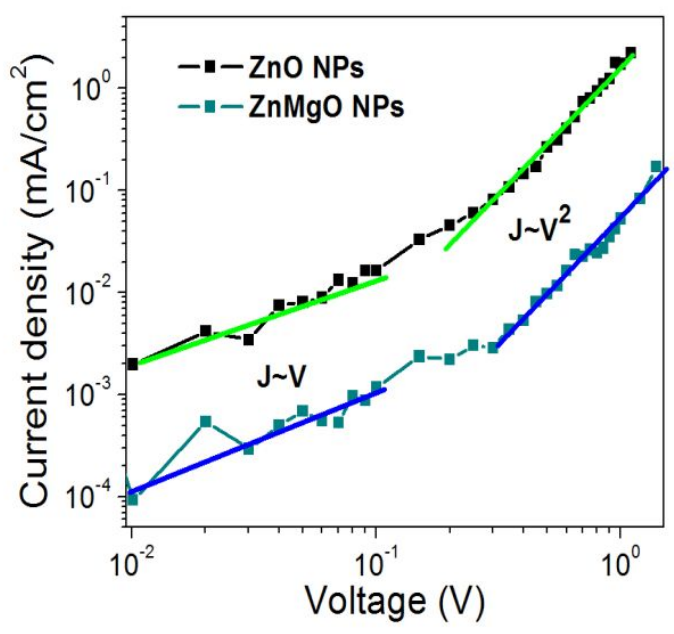

Figure S6. Current density-voltage (J-V) characteristic of the electron-only device with a structure of Al/NPs $(40 \mathrm{~nm}) / \mathrm{Al}$.

The $J-V$ curve clearly displays two regions, i.e. the ohmic region $(J \sim V)$ and space charge limited current (SCLC) region $\left(J \sim V^{2}\right)$. The electron mobility $\mu_{\mathrm{e}}$ of the $\mathrm{ZnO}$ NP films can be obtained by fitting the SCLC region according to Child's law ${ }^{4-5}$ $J=9 / 8 \cdot \varepsilon_{r} \varepsilon_{0} \mu_{e} \cdot V^{2} / \mathrm{d}^{3}$, where $\varepsilon_{\mathrm{r}}$ and $\varepsilon_{0}$ are relative and vacuum permittivity, respectively, and $d$ is the film thickness. By assuming $\varepsilon_{\mathrm{r}}=4$, the fitting leads to a zero field electron mobility $\mu_{\mathrm{e}}$ of $2.78 \times 10^{-4} \mathrm{~cm}^{2} \mathrm{~V}^{-1} \mathrm{~s}^{-1}$ for $\mathrm{ZnO}$ NPs and $0.36 \times 10^{-5} \mathrm{~cm}^{2} \mathrm{~V}^{-1} \mathrm{~s}^{-1}$ for $\mathrm{ZnMgO} \mathrm{NPs}$, respectively. 
a

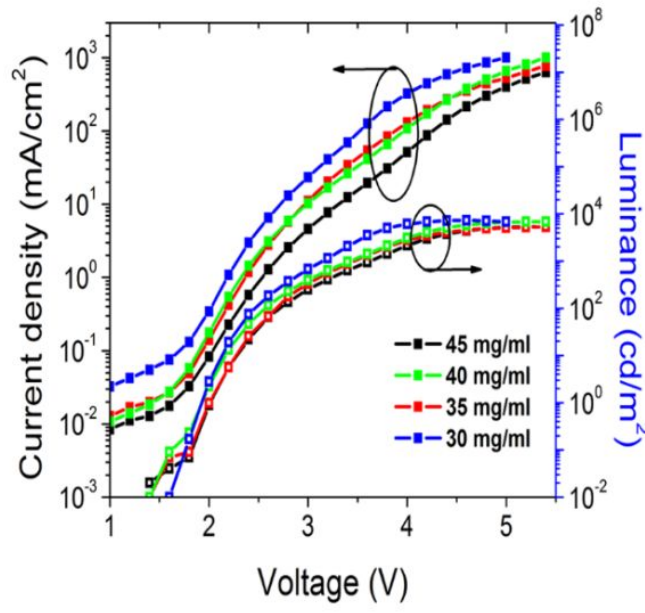

b

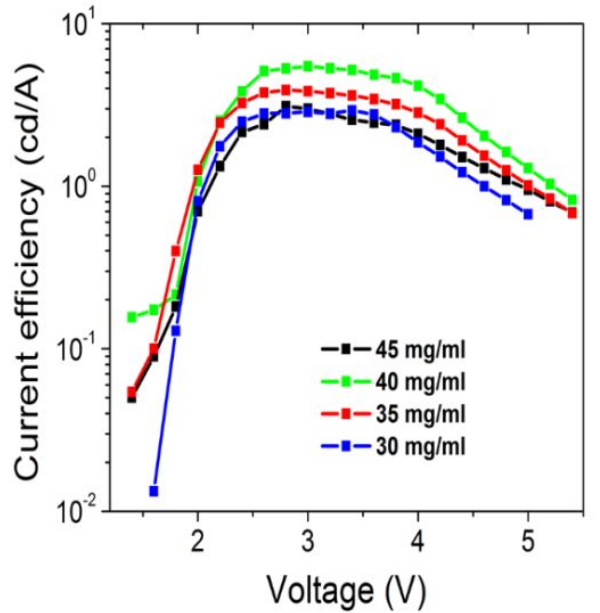

Figure S7. EL performances of QLEDs with different spin-coating speed of QD layer.

(a) Luminance-voltage-current density (L-V-J) characteristics of QLEDs with or without (w/o) $\mathrm{ZnMgO}$ layer. (b) $\mathrm{CE}$ properties properties as a function of voltage.

a

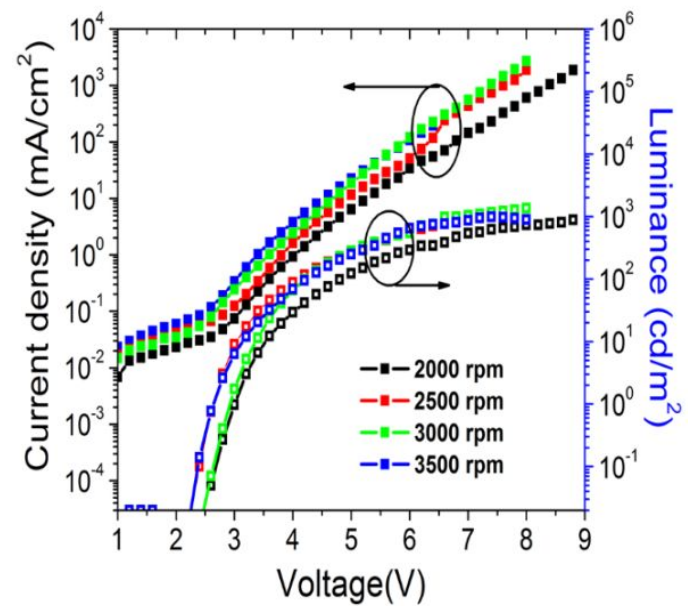

b

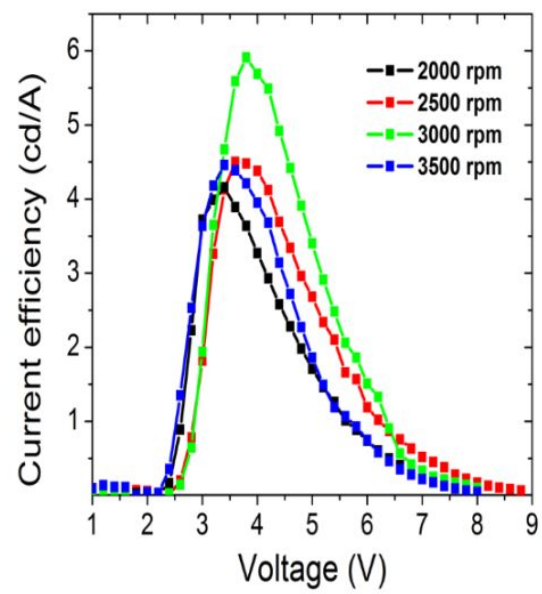

Figure S8. EL performances of QLEDs with different concentration of $\mathrm{ZnMgO} \mathrm{NP}$ layer. (a) Luminance-voltage-current density (L-V-J) characteristics of QLEDs with or without (w/o) $\mathrm{ZnMgO}$ layer. (b) $\mathrm{CE}$ properties properties as a function of voltage. 


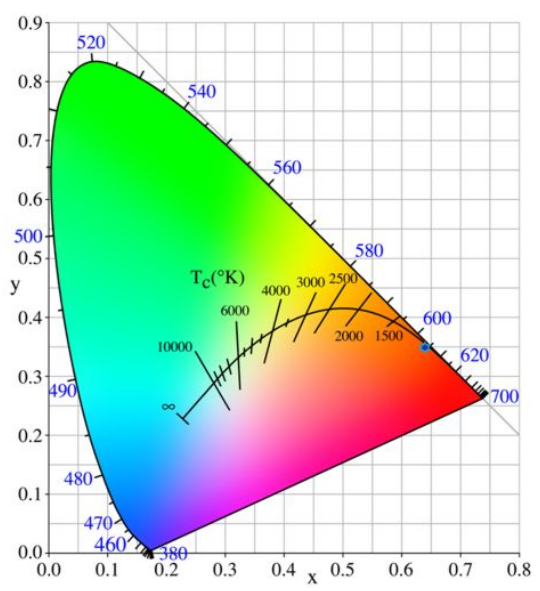

Figure S9. CIE property of device C.

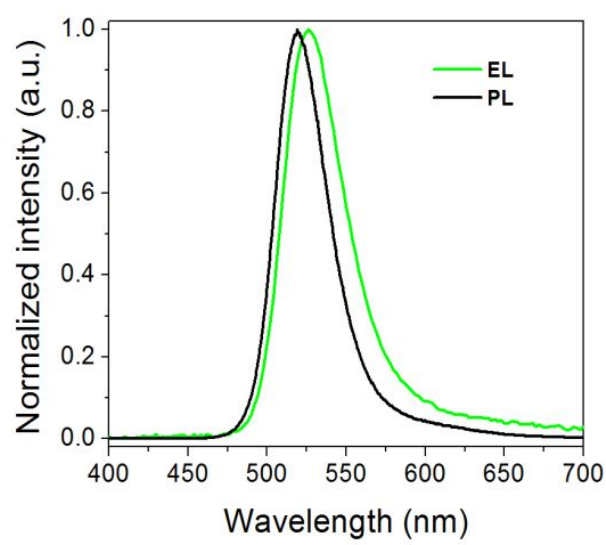

Figure S10. Normalized PL and EL spectra of green QLED.

The peak wavelength of EL spectra is $540 \mathrm{~nm}$. The full width at half maximum (FWHM) is $44 \mathrm{~nm}$. Due to electric-field-induce Stark effect and the interdot interactions, the EL spectra have $10 \mathrm{~nm}$ red-shift and a broadened FWHM compared with that of PL spectra (@ 530 nm and FWHM of 39 nm). 
a

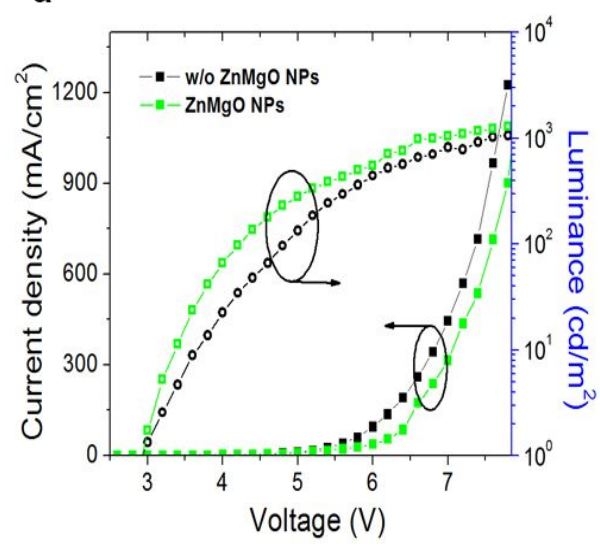

b

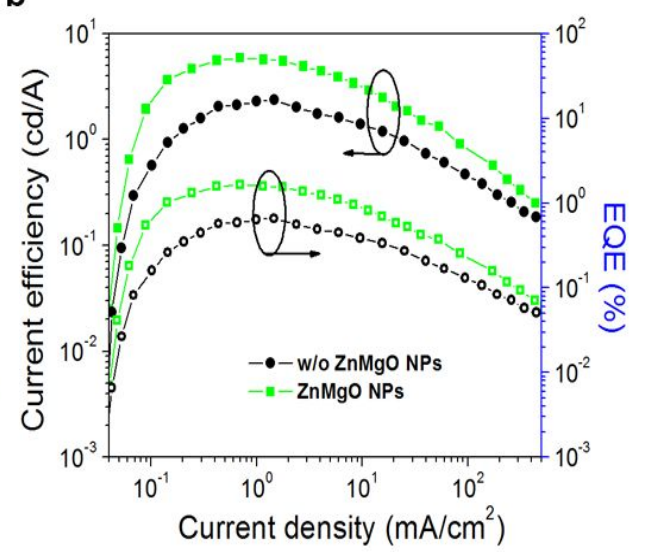

Figure S11. EL performances of green QLEDs. (a) Luminance-voltage-current density (L-V-J) characteristics of QLEDs. (b) CE properties and EQE properties as a function of current density.

Table S4. Summary of maximum luminance $\left(\mathrm{L}_{\max }\right)$, maximum $\mathrm{CE}\left(\mathrm{CE}_{\max }\right)$, maximum $\mathrm{EQE}\left(\mathrm{EQE}_{\max }\right)$ of green device with (w/) or without (w/o) ZnMgO NPs layer.

\begin{tabular}{cccc}
\hline Device & $\mathbf{L}_{\text {max }}$ & $\mathbf{C E}_{\max }$ & $\mathbf{E Q E}_{\mathbf{m a x}}$ \\
& $\mathbf{( c d / \mathbf { m } ^ { 2 } )}$ & $\mathbf{( c d / A )}$ & $\mathbf{( \% )}$ \\
\hline $\mathbf{w} /$ & 1360 & 5.91 & 1.65 \\
$\mathbf{w} / \mathbf{0}$ & 1060 & 2.36 & 0.66 \\
\hline
\end{tabular}

Table S5. Summary of EL performance: device structure, QD, PL QY, peak wavelength $\left(\lambda_{\max }\right)$, maximum $\mathrm{CE}$, maximum $\mathrm{EQE}$ and publication year of reported InP QLEDs and this work. 


\begin{tabular}{|c|c|c|c|c|c|c|c|}
\hline Device & Structure & QDs & $\begin{array}{l}\text { PL QY } \\
(\%)\end{array}$ & $\begin{array}{l}\lambda_{\max } \\
(\mathbf{n m})\end{array}$ & $\begin{array}{c}\mathrm{CE} \\
(\mathrm{cd} / \mathrm{A})\end{array}$ & $\begin{array}{c}\text { EQE } \\
(\%)\end{array}$ & year \\
\hline Blue & $\begin{array}{c}\text { ITO/PEDOT:PSS/TFB/ } \\
\text { QDs/ZnO/Al }\end{array}$ & $\mathrm{InP} / \mathrm{GaP} / \mathrm{ZnS} / / \mathrm{ZnS}$ & 81 & 488 & I & 1.01 & $2020^{6}$ \\
\hline \multirow{7}{*}{ Green } & $\begin{array}{c}\mathrm{ITO} / \mathrm{ZnO} / \mathrm{PFN} / \mathrm{QDs} / \mathrm{TC} \\
\mathrm{TA} / \mathrm{MoO}_{3} / \mathrm{Al}\end{array}$ & $\mathrm{InP} / \mathrm{ZnSeS}$ & $>70$ & 518 & 10.9 & 3.46 & $2013^{7}$ \\
\hline & $\begin{array}{c}\mathrm{ITO} / \mathrm{ZnO} / \mathrm{QDs} / \mathrm{CBP} / \mathrm{Mo} \\
\mathrm{O}_{3} / \mathrm{Al}\end{array}$ & $\mathrm{InP} / \mathrm{ZnSe} / \mathrm{ZnS}$ & I & $\sim 550$ & 1.892 & l & $2015^{8}$ \\
\hline & $\begin{array}{c}\text { AgNW-cPI } \\
\text { composite/poly-TPD/ } \\
\text { QDs/TPBi/LiF/Al }\end{array}$ & $\mathrm{InP} / \mathrm{ZnSe} / \mathrm{ZnS}$ & / & 540 & 8.6 & l & $2017^{9}$ \\
\hline & $\begin{array}{c}\mathrm{ITO} / \mathrm{ZnMgO} / \mathrm{QD} / \mathrm{TcTa} / \\
\text { NPB/HAT-CN/Al }\end{array}$ & $\mathrm{InP} / \mathrm{ZnSeS} / \mathrm{ZnS}$ & 70 & 545 & 4.44 & l & 201710 \\
\hline & $\begin{array}{c}\text { ITO/PEDOT:PSS/TFB/ } \\
\text { QDs/ZnO/Al }\end{array}$ & $\mathrm{InP} / \mathrm{GaP} / \mathrm{ZnS}$ & 67 & 530 & 13.7 & 6.3 & $2019^{11}$ \\
\hline & $\begin{array}{c}\text { ITO/PEDOT:PSS/poly- } \\
\text { TPD/PVK/QDs/ } \\
\mathrm{ZnMgO/Al}\end{array}$ & $\mathrm{InP} / \mathrm{ZnS}$ & I & 530 & l & 13.6 & $2019^{12}$ \\
\hline & $\begin{array}{l}\text { ITO/PEDOT:PSS/TFB/ } \\
\text { QDs/ ZnMgO/ZnO/Al }\end{array}$ & $\mathrm{InP} / \mathrm{ZnS}$ & 40 & 526 & 5.91 & 1.65 & This work \\
\hline $\begin{array}{l}\text { Yellow- } \\
\text { orange }\end{array}$ & $\begin{array}{c}\text { ITO/PEDOT:PSS/poly- } \\
\text { TPD/QDs/ZnO/Al }\end{array}$ & $\mathrm{InP} / \mathrm{ZnO}$ & I & 585 & 1 & 0.53 & $2019^{13}$ \\
\hline \multirow{9}{*}{ Red } & $\begin{array}{c}\mathrm{ITO} / \mathrm{ZrO}_{2} / \mathrm{QDs} / \mathrm{TAPC} / \\
\mathrm{MoO}_{3} / \mathrm{Al}\end{array}$ & $\mathrm{InP} / \mathrm{ZnSe} / \mathrm{ZnS}$ & I & 600 & 0.47 & l & $2016^{14}$ \\
\hline & $\begin{array}{c}\text { ITO/PEDOT:PSS/PVK } \\
\text { or TFB/ QDs/ZnO } \\
\text { NPs/Al }\end{array}$ & $\mathrm{InP} / \mathrm{ZnSeS} / \mathrm{ZnS}$ & 82 & 614 & 4.2 & 2.5 & $2016^{15}$ \\
\hline & $\begin{array}{c}\mathrm{ITO} / \mathrm{ZnO} / \mathrm{QDs} / \mathrm{CBP} / \mathrm{Mo} \\
\mathrm{O}_{3} / \mathrm{Al}\end{array}$ & $\mathrm{InP} / \mathrm{ZnSe} / \mathrm{ZnS}$ & 73 & 607 & 13.6 & 6.3 & $2018^{16}$ \\
\hline & $\begin{array}{l}\text { ITO/PEDOT:PSS/poly- } \\
\text { TPD/QDs/ZnMgO/Ag }\end{array}$ & $\mathrm{InP} / \mathrm{ZnSe} / \mathrm{ZnS}$ & $>90$ & 630 & l & 12.2 & $2019^{17}$ \\
\hline & $\begin{array}{c}\text { ITO/PEDOT:PSS/TFB/ } \\
\text { QDs/ZnMgO/Ag }\end{array}$ & $\mathrm{InP} / \mathrm{ZnSe} / \mathrm{ZnS}$ & $\sim 100$ & 630 & l & 21.4 & $2019^{18}$ \\
\hline & $\begin{array}{c}\text { ITO/PEDOT:PSS/TFB/In } \\
\text { P-RQD/ZnMgO/Al }\end{array}$ & $\mathrm{InP} / \mathrm{ZnSe} / \mathrm{ZnS}$ & 60 & / & 11.6 & l & $2019^{19}$ \\
\hline & $\begin{array}{c}\text { ITO/PEDOT:PSS/TFB/ } \\
\text { QDs/doped-ZnO/Al }\end{array}$ & $\mathrm{InP} / \mathrm{ZnS}$ & 54 & 633 & 3.59 & 4.24 & $2019^{20}$ \\
\hline & $\begin{array}{c}\mathrm{ITO} / \mathrm{ZnO} / \mathrm{PEI} / \mathrm{QD} / \mathrm{TPBi} / \\
\mathrm{CBP} / \mathrm{MoO}_{3} / \mathrm{Al}\end{array}$ & $\mathrm{InP} / \mathrm{ZnS}$ & 32 & 610 & 8.54 & 6.63 & $2020^{21}$ \\
\hline & $\begin{array}{l}\text { ITO/PEDOT:PSS/TFB/ } \\
\text { QDs/ ZnMgO/ZnO/Al }\end{array}$ & $\mathrm{InP} / \mathrm{ZnS}$ & 45 & 620 & 5.46 & 3.56 & This work \\
\hline
\end{tabular}




\section{References}

(1) Kwak, J.; Bae, W. K.; Lee, D.; Park, I.; Lim, J.; Park, M.; Cho, H.; Woo, H.; Yoon do, Y.; Char, K.; Lee, S.; Lee, C., Bright and efficient Full-Color Colloidal Quantum Dot Light-Emitting Diodes Using an Inverted Device Structure. Nano Lett. 2012, 12, 2362-2366.

(2) Wang, L.; Lv, Y.; Lin, J.; Fan, Y.; Zhao, J.; Wang, Y.; Liu, X., High-Efficiency Inverted Quantum Dot Light-Emitting Diodes with Enhanced Hole Injection. Nanoscale 2017, 9, 6748-6754..

(3) Kim, J. H.; Han, C. Y.; Lee, K. H.; An, K. S.; Song, W.; Kim, J.; Min, S. O.; Do, Y. R.; Yang, H., Performance Improvement of Quantum Dot-Light-Emitting Diodes Enabled by an Alloyed $\mathrm{ZnMgO}$ Nanoparticle Electron Transport Layer. Chem. Mater. 2015, 27, 197-204.

(4) Qian, L.; Zheng, Y.; Xue, J.; Holloway, P. H., Stable and Efficient Quantum-Dot Light-Emitting Diodes Based on Solution-Processed Multilayer Structures. Nat. Photon. 2011, 5, 543-548.

(5) Lampert, M. A., Simplified Theory of Space-Charge-Limited Currents in an Insulator with Traps. Phys. Rev. 1956, 103, 1648.

(6) Zhang, H.; Ma, X.; Lin, Q.; Zeng, Z.; Wang, H.; Li, L. S.; Shen, H.; Jia, Y.; Du, Z., High-Brightness Blue InP Quantum Dot-Based Electroluminescent Devices: The Role of Shell Thickness. J. Phys. Chem. Lett. 2020, 11, 960-967.

(7) Jaehoon, L.; Myeongjin, P.; Ki, B. W.; Donggu, L.; Seonghoon, L.; Changhee, L.; Kookheon, C. Highly Efficient Cadmium-free Quantum Dot Light-emitting Diodes Enabled by the Direct Formation of Excitons within InP@ZnSeS Quantum Dots. ACS Nano 2013, 7, 9019.

(8) Jang, I.; Kim, J.; Ippen, C.; Greco, T.; Oh, M. S.; Lee, J.; Kim, W. K.; Wedel, A.; Han, C. J.; Park, S. K. Inverted InP Quantum Dot Light-emitting Diodes using Low-temperature Solution-processed Metal-oxide as an Electron Transport Layer. Jpn. J. Appl. Phys. 2015, $54,02 \mathrm{BC} 01$.

(9) Kim, J.-W.; Kim, J. Flexible InP based Quantum Dot Light-emitting Diodes using Ag Nanowire-colorless Polyimide Composite Electrode. J. Vac. Sci. Technol., B: Nanotechnol. Microelectron.: Mater., Process., Meas., Phenom. 2017, 35, 04E101.

(10) Wang, H. C.; Zhang, H.; Chen, H. Y.; Yeh, H. C.; Tseng, M. R.; Chung, R. J.; Chen, S.; Liu, R. S. Cadmium-Free InP/ZnSeS/ZnS Heterostructure-Based Quantum Dot Light-Emitting Diodes with a $\mathrm{ZnMgO}$ Electron Transport Layer and a Brightness of Over $10000 \mathrm{~cd} \mathrm{~m}-2$. Small 2017, 13, 1603962.

(11) Zhang, H.; Hu, N.; Zeng, Z.; Lin, Q.; Zhang, F.; Tang, A.; Jia, Y.; Li, L. S.; Shen, H.; Teng, F.; Du, Z. High - Efficiency Green InP Quantum Dot - Based Electroluminescent Device Comprising Thick - Shell Quantum Dots. Adv. Opt. Mater. 2019, 7, 1801602.

(12) Moon, H.; Lee, W.; Kim, J.; Lee, D.; Cha, S.; Shin, S.; Chae, H. Composition-tailored $\mathrm{ZnMgO}$ Nanoparticles for Electron Transport Layers of Highly Efficient and Bright InP-based Quantum Dot Light Emitting Diodes. Chem. Commun. 2019, 55, 13299-13302.

(13) Karatum, O.; Jalali, H. B.; Sadeghi, S.; Melikov, R.; Srivastava, S. B.; Nizamoglu, S. Light-Emitting Devices Based on Type-II InP/ZnO Quantum Dots. ACS Photonics 2019, 
6, 939-946.

(14) Kim, H. Y.; Park, Y. J.; Kim, J.; Han, C. J.; Lee, J.; Kim, Y.; Greco, T.; Ippen, C.; Wedel, A.; Ju, B.-K.; Oh, M. S. Transparent InP Quantum Dot Light-Emitting Diodes with ZrO2 Electron Transport Layer and Indium Zinc Oxide Top Electrode. Adv. Funct. Mater. 2016, 26, 3454-3461.

(15) Jo, J. H.; Kim, J. H.; Lee, K. H.; Han, C. Y.; Jang, E. P.; Do, Y. R.; Yang, H. High-efficiency Red Electroluminescent Device based on Multishelled InP Quantum Dots. Opt. Lett. 2016, 41, 3984-3987.

(16) Cao, F.; Wang, S.; Wang, F.; Wu, Q.; Zhao, D.; Yang, X. A Layer-by-Layer Growth Strategy for Large-Size InP/ZnSe/ZnS Core-Shell Quantum Dots Enabling High-Efficiency Light-Emitting Diodes. Chem. Mater. 2018, 30, 8002-8007.

(17) Li, Y.; Hou, X.; Dai, X.; Yao, Z.; Lv, L.; Jin, Y.; Peng, X. Stoichiometry-Controlled InP-Based Quantum Dots: Synthesis, Photoluminescence, and Electroluminescence. $J$. Am. Chem. Soc. 2019, 141, 6448-6452.

(18) Won, Y.-H.; Cho, O.; Kim, T.; Chung, D.-Y.; Kim, T.; Chung, H.; Jang, H.; Lee, J.; Kim, D.; Jang, E. Highly Efficient and Stable InP/ZnSe/ZnS Quantum Dot Light-emitting Diodes. Nature 2019, 575, 634-638.

(19) Li, D.; Kristal, B.; Wang, Y.; Feng, J.; Lu, Z.; Yu, G.; Chen, Z.; Li, Y.; Li, X.; Xu, X. Enhanced Efficiency of InP-Based Red Quantum Dot Light-Emitting Diodes. ACS Appl. Mater. Interfaces 2019, 11, 34067-34075.

(20) Chen, F.; Lv, P.; Li, X.; Deng, Z.; Teng, F.; Tang, A. Highly-efficient and All-solution-processed Red-emitting InP/ZnS-based Quantum-dot light-emitting Diodes Enabled by Compositional Engineering of Electron Transport Layers. J. Mater. Chem. C 2019, 7, 7636-7642.

(21) Wang, Y.; Chen, Z.; Wang, T.; Zhang, H.; Zhang, H.; Wang, R.; Ji, W. Efficient Structure for InP/ZnS-Based Electroluminescence Device by Embedding the Emitters in the Electron-Dominating Interface. J. Phys. Chem. Lett. 2020, 11, 1835-1839. 
